\title{
SETCOMP - Sistema Especialista para Avaliação Ergonômica do Posto de Trabalho com uso do Computador
}

\author{
James Marcelo Schulz, Roberta Francine Schmachtenberg, Rejane Frozza, Marcia \\ Elena Jochims Kniphoff da Cruz
}

\author{
Departamento de Computação \\ Curso de Computação Licenciatura \\ Universidade de Santa Cruz do Sul (UNISC) \\ Av. Independência, 2293 - 96815-900 - Santa Cruz do Sul, RS \\ jamesschulz@mx2.unisc.br, robertafs@mx2.unisc.br, frozza@unisc.br, \\ mcruzeunisc.br
}

\begin{abstract}
This paper reports the construction of an expert system (ES) for the ergonomic evaluation of the computer workstation, called SETCOMP. The ES was developed with support of the tool Expert SINTA, which uses Artificial Intelligence techniques for logical reasoning. Between the stages of the construction of the ES, we highlight the knowledge representation, which enables the modeling of human knowledge to a computational knowledge base. The results describe the ES validation process. The conclusion discusses the facilities and difficulties faced by the authors/developers.
\end{abstract}

Resumo. Este artigo relata o processo de desenvolvimento de um Sistema Especialista (SE) para avaliação ergonômica do posto de trabalho com uso do computador, denominado SETCOMP. O SE foi desenvolvido com auxílio da ferramenta Expert SINTA, que utiliza técnicas de Inteligência Artificial para o raciocínio lógico. Entre as etapas de desenvolvimento do SE, se destaca a etapa de representação do conhecimento, que viabiliza a modelagem do conhecimento humano para uma base de conhecimento computacional. Os resultados descrevem o processo de validação do SE. A conclusão discute as facilidades e dificuldades enfrentadas pelos autores/desenvolvedores.

\section{Introdução}

A área de Tecnologia da Informação (TI) proporcionou um crescimento exponencial na venda de computadores de uso pessoal (personal computers). O computador se tornou um aliado indispensável no atual mundo de trabalho e nos ambientes educacionais. No entanto, o uso prolongado das máquinas suscita discussões sobre a utilização de ergonomia no planejamento de tais ambientes e na definição de padrões e normas regulamentadoras. Entende-se como ergonomia "o conjunto de conhecimentos científicos relativos ao homem e necessários para a concepção de ferramentas, máquinas e dispositivos que possam ser utilizados com o máximo de conforto, segurança e eficácia" (Wisner, 1987). Desta forma, este trabalho tem por objetivo relatar o processo de desenvolvimento de um Sistema Especialista (SE) capaz de auxiliar Técnicos de Segurança do Trabalho e gestores empresariais/educacionais na tomada de decisão quanto à condição ergonômica de colaboradores, estudantes e professores no posto de trabalho com uso do computador. 
VIII Congresso Brasileiro de Informática na Educação (CBIE 2019)

Anais do XXV Workshop de Informática na Escola (WIE 2019)

Atualmente, este tipo de avaliação contempla a Norma Regulamentadora 17 (NR 17), de 2002. A NR 17 estabelece parâmetros que permitem a adaptação das condições de trabalho às características psicofisiológicas dos trabalhadores, de modo a proporcionar um máximo de conforto, segurança e desempenho eficiente. Também cabe ao empregador realizar a análise ergonômica do trabalho, devendo a mesma abordar, no mínimo, as condições de trabalho, conforme estabelecido na Norma Regulamentadora (NR 17, 2002).

Devido à informatização, os postos de trabalho se tornaram presentes em praticamente todas as profissões. Em alguns casos, o computador é utilizado esporadicamente; em outros, o usuário passa horas com o corpo quase estático, com a atenção fixa na tela do monitor e as mãos sobre o teclado e mouse, realizando operações de digitação, altamente repetitivas (Iida, 2005). Neste último caso, os usuários podem sofrer danos a sua saúde, se questões de ergonomia não forem adotadas e colocadas em prática.

A Inteligência Artificial está associada ao conceito de conhecimento, como tecnologia chave para o desenvolvimento de softwares do futuro. O computador, diferente da mente humana, não pode ter experiências e nem aprender. Entretanto, os Sistemas Inteligentes propõem a utilização do conhecimento fornecido por especialistas humanos para o desenvolvimento de programas que reproduzem o raciocínio e a inteligência humana, com o objetivo de resolver problemas e auxiliar na tomada de decisão.

Em sistemas inteligentes, destaca-se: habilidades para usar conhecimento para desempenhar tarefas ou resolver problemas e a capacidade de trabalhar com problemas complexos com aplicações reais e podem ser desenvolvidos com o uso de técnicas, como: Aquisição de Conhecimento, Aprendizado de Máquina, Redes Neurais, Lógica Fuzzy, Computação Evolutiva, Agentes e Multiagentes, Mineração de Dados, Mineração de Textos, Redes Bayesianas, Sistemas Especialistas, Raciocínio Baseado em Casos, entre outros (REZENDE, 2003).

Nesse contexto, o artigo está organizado da seguinte forma: a seção 2 descreve o domínio da aplicação do SETCOMP; a seção 3 descreve as etapas de desenvolvimento do SETCOMP; a seção 4 descreve os resultados obtidos por meio da validação do sistema; a seção 5 conclui o trabalho.

\section{Métodos}

Para o desenvolvimento do sistema especialista, dois processos foram essenciais: o de aquisição do conhecimento e o de representação do conhecimento.

Segundo Wermuth (2007) a aquisição do conhecimento é considerada uma das fases mais complexas do processo de desenvolvimento de um SE, por se tratar da interação entre o agente humano, responsável por desenvolver o sistema (engenheiro do conhecimento) e a fonte humana de conhecimento (o especialista).

A aquisição do conhecimento se traduz como o processo de extração do conhecimento do especialista humano em seu domínio de competência. O conhecimento pode ser factual (advindo de livros, revistas, artigos e outros) e heurístico (advindo das vivências e experiências dos especialistas). Nesta etapa do processo, o engenheiro do conhecimento tem a função de captar o raciocínio do especialista para saber como ele 
VIII Congresso Brasileiro de Informática na Educação (CBIE 2019)

Anais do XXV Workshop de Informática na Escola (WIE 2019)

resolve o problema e traduzi-lo para um sistema computacional, capaz de tomar decisões, para depois modelar a base de conhecimento (Weber, 1993).

A representação do conhecimento é uma forma de modelagem do conhecimento humano para conhecimento armazenado em máquina (Wermuth, 2007). Nessa etapa do processo ocorre a formalização e organização de conceitos (mecanismos de inferência) obtidos com o especialista humano. Conforme Rezende (2003) a representação do conhecimento deve ser compreensível ao ser humano, abstraindo-se dos detalhes de como funciona internamente o processador de conhecimento, permitindo que o próprio especialista humano consiga avaliá-la e interpretá-la.

Existem inúmeras técnicas de representação do conhecimento. Dentre elas, se destacam: regras de produção, redes semânticas e frames. A arquitetura mais comum de SEs é a que envolve regras de produção. Essas regras são um conjunto de condições (SE - ENTÃO), com a possibilidade de inclusão de conectivos lógicos relacionando os atributos no escopo do conhecimento e o uso de probabilidades.

\section{Resultados e discussão}

Esta seção descreve as etapas de desenvolvimento do SE proposto.

\subsection{Etapa 1: representação do conhecimento}

A primeira etapa abrangeu o processo de representação do conhecimento (RC) contido na NR 17 em relação aos aspectos a serem avaliados da ergonomia no posto de trabalho com uso do computador. O documento estabelece os parâmetros que permitem a adaptação das condições de trabalho às características psicofisiológicas dos trabalhadores, de modo a proporcionar um máximo de conforto, segurança e desempenho eficiente (NR 17, 2002).

O conhecimento do domínio foi representado por regras de produção. As regras completas do SE podem ser acessadas através do seguinte endereço: <bit.ly/SETCOMP$\mathrm{RP}>{ }^{1}$. O SETCOMP compreende o total de 39 regras.

\subsection{Etapa 2: Implementação do SE no Expert SINTA}

A etapa de representação do conhecimento do SETCOMP foi fundamental para a etapa de implementação do SE com o uso da ferramenta Expert SINTA, uma vez que a ferramenta utiliza um modelo de representação do conhecimento baseado em regras de produção e probabilidade. A Figura 1 ilustra uma regra de produção implementada no Expert SINTA.

\footnotetext{
${ }^{1}$ Endereço original: $<$ https://docs.google.com/document/d/1kJuo6OLK3MfiHannxzWuxAN4HIGjJxL46oEf0Rwdjsw/edit?us $\mathrm{p}=$ sharing $>$.
} 
VIII Congresso Brasileiro de Informática na Educação (CBIE 2019)

Anais do XXV Workshop de Informática na Escola (WIE 2019)

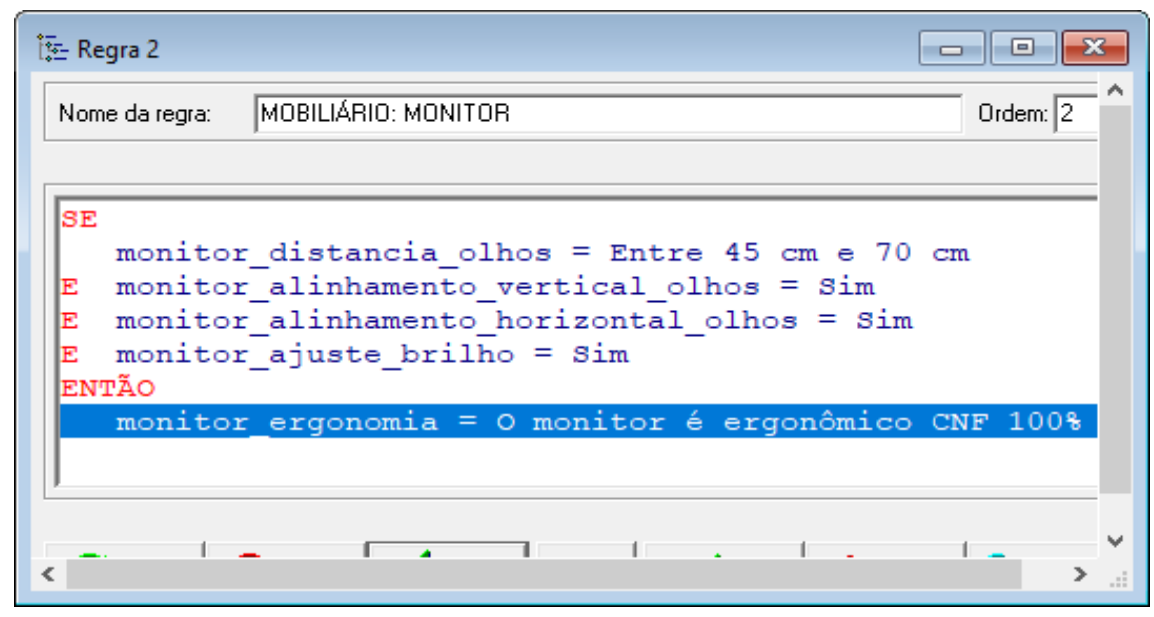

Figura 1. Regra de produção no Expert SINTA

\subsection{Interação do usuário com o sistema}

Ao iniciar o sistema, o usuário deve responder perguntas relacionadas ao mobiliário e ao ambiente de trabalho que está inserido.

O número de perguntas exibidas em interface pode variar conforme as respostas informadas pelo usuário. Por exemplo, se na primeira questão referente ao item "cadeira", o usuário dispuser de uma resposta contrária à característica de uma cadeira ergonômica, o sistema encerra as questões referentes ao item, pois conclui que a cadeira do usuário não é ergonômica. Caso contrário, continuará com as questões relacionadas ao item.

Em um posto de trabalho $0 \%$ ergonômico, por exemplo, o sistema irá solicitar o máximo de seis perguntas. Em um posto de trabalho 100\% ergonômico, o sistema irá solicitar entre 14 e 15 perguntas.

Como forma de validação do SETCOMP, foram coletadas informações do sistema com cinco colaboradores convidados a utilizá-lo para avaliação do seu posto de trabalho.

\subsection{Descrição da validação}

Após a interação dos colaboradores com o sistema, foi realizada a validação através de um formulário de avaliação.

O formulário abrangeu as questões dispostas no Quadro 1. O Quadro 1 está dividido em duas colunas: a coluna 1 abrange as perguntas dispostas no formulário e as opções de respostas para cada pergunta; a coluna 2 abrange as respostas informadas pelos 5 colaboradores.

\begin{tabular}{|l|l|}
\hline \multicolumn{1}{|c|}{ Pergunta } & \multicolumn{1}{|c|}{ Respostas } \\
\hline $\begin{array}{l}\text { 1. Na sua opinião, este sistema pode ser utilizado por profissionais } \\
\text { da área de Segurança do Trabalho ou gestor em um contexto } \\
\text { organizacional? } \\
\text { * Sim, Não, Não sei }\end{array}$ & $\begin{array}{l}\text { Colaborador 1, 2, 3 e 5: Sim } \\
\text { Colaborador 4: Não sei }\end{array}$ \\
\hline $\begin{array}{l}\text { 2. Na sua opinião, as questões contemplaram os itens de ergonomia } \\
\text { relacionados ao posto de trabalho com uso do computador? } \\
\text { * Sim, Não, Não sei }\end{array}$ & Colaborador 1, 2, 3, 4 e 5: Sim \\
\hline
\end{tabular}


VIII Congresso Brasileiro de Informática na Educação (CBIE 2019)

Anais do XXV Workshop de Informática na Escola (WIE 2019)

\begin{tabular}{|c|c|}
\hline $\begin{array}{l}\text { 3. As questões foram de fácil entendimento? } \\
\text { * Sim, Não, Não sei }\end{array}$ & Colaborador $1,2,3,4$ e $5:$ Sim \\
\hline $\begin{array}{l}\text { 4. As questões foram extensas (exigiram bastante tempo de } \\
\text { leitura?) } \\
\text { * Sim, Não, Não sei }\end{array}$ & Colaborador $1,2,3,4$ e 5: Não \\
\hline $\begin{array}{l}\text { 5. Em média, quanto tempo você demorou para responder às } \\
\text { questões solicitadas pelo sistema? }\end{array}$ & $\begin{array}{l}\text { Colaborador } 1 \text { e } 5: 1,5 \text { minuto } \\
\text { Colaborador } 2 \text { e } 3: 1 \text { minuto } \\
\text { Colaborador } 4: 2 \text { minutos }\end{array}$ \\
\hline $\begin{array}{l}\text { 6. Você considera que o diagnóstico gerado pelo SETCOMP, a } \\
\text { respeito do índice }(\%) \text { de qualidade das condições do seu local de } \\
\text { trabalho, foi coerente? }\end{array}$ & Colaborador $1,2,3,4$ e $5:$ Sim \\
\hline $\begin{array}{l}\text { 7. Qual foi o índice }(\%) \text { de qualidade das condições de trabalho que } \\
\text { o sistema gerou referente ao seu local de trabalho? }\end{array}$ & $\begin{array}{l}\text { Colaborador } 1 \text { e } 4: 66,6 \% \\
\text { Colaborador } 2,3 \text { e } 5: 50 \%\end{array}$ \\
\hline
\end{tabular}

\section{Quadro 1. Resultados da validação do SETCOMP}

\section{Conclusão}

Este trabalho relatou o processo de desenvolvimento de um Sistema Especialista (SE) capaz de auxiliar Técnicos de Segurança do Trabalho e gestores empresariais/educacionais na tomada de decisão quanto à ergonomia de colaboradores, estudantes e professores no posto de trabalho com uso do computador.

Como proposta futura, pretende-se validar o sistema com um especialista humano do domínio de competência, Engenheiro ou Técnico em Segurança do Trabalho. O Sistema Especialista SETCOMP pode ser acessado através do seguinte endereço: $<$ bit.ly/SETCOMP-sistema $>^{2}$.

\section{Referências}

Associação Brasileira de Ergonomia (ABERGO). O que é ergonomia. Disponível em: <http://www.abergo.org.br/internas.php?pg=o_que_e_ergonomia>. Disponível em: 04 maio 2019.

NR 17 - Ergonomia. Ministério do Trabalho e Emprego. Brasília: Ministério do Trabalho e Emprego, 2002. Disponível em: 〈http://www.guiatrabalhista.com.br/legislacao/nr/nr17.htm>. Acesso em: 04 maio 2019.

Iida, Itiro; BUARQUE, Lia. Ergonomia: projeto e produção. Editora Blucher, 2016.

Rezende, Solange. O. Sistemas Inteligentes: fundamentos e aplicações. Barueri, São Paulo: Manole, 2003.

Wermuth, Silvia Letícia Pozzebon. Modelagem matemática e computacional de um sistema de apoio à decisão na indústria. 2007. Disponível em: <https://repositorio.unisc.br/jspui/handle/11624/310>. Acesso em: 01 maio 2019.

Weber, Rosina de Oliveira et al. Sistema especialista difuso para análise de crédito. 1993.

Wisner, Allan. Por dentro do trabalho. Ergonomia: método e técnica. São Paulo, FTD/Oboré. 1987. 4. In: II Workshop Gestão Integrada: Risco e Sustentabilidade, São Paulo.

\footnotetext{
${ }^{2}$ Endereço original: 〈https://drive.google.com/open?id=1AOBkXFlu6SJrUnoyGiitxuDIYDJdFnsf>.
} 